\title{
BIOMECHANICAL FACTORS IN THE PROGRESSION OF IDIOPATHIC SCOLIOSIS
}

\author{
Albert B. Schultz \\ Department of Mechanical Engineering and Applied Mechanics \\ University of Michigan \\ Ann Arbor, Michigan
}

\begin{abstract}
Idiopathic scoliosis is present when, in upright positions of the trunk, the spine curves to the side for unknown reasons. This paper reviews evidence concerning some biomechanical factors that might underlie the progression of such curves. The review concentrates on studies conducted in our laboratories. Arguments are made, based on biomechanical analyses and experiments, that progression occurs because of defects in the postural control system of the spine.
\end{abstract}

Keywords - Idiopathic scoliosis, Progression mechanics, Myoelectric activity.

\section{INTRODUCTION}

In upright postures of the trunk of humans who have structurally-normal spines, the vertebrae when viewed from the front or back lie along a nearly straight vertical line. If the vertebrae form one or more curves to the side, a case of scoliosis exists.

If not treated, most scoliosis curves will stabilize, but a few will progress; that is, get worse. Lateral curves in the spine tend to become clinically significant just at the onset of the rapid growth period of adolescence. Mild scoliosis curves result in cosmetic deformities of the trunk. These create psychosocial problems for the children who have them. Severe scoliosis curves result in trunk deformities of magnitudes that eventually compromise the internal viscera and thus become life-threatening. Most cases of scoliosis have no know cause; these are called idiopathic cases.

Regarding the prevalence of scoliosis, Shands and Eisberg (11) found that one person in 200 has a scoliotic curve of 20 degrees or more. Lateral curves of 10 degrees or more were found by Shands and Eisberg in almost $2 \%$ of the population they studied. Brooks et al. (1), on screening 3500 seventh- and eighth-grade students, found a $13.6 \%$ prevalence of measurable scoliotic

Acknowledgement-The support of Public Health Service Grants AM 15575 and AM 33948 for this research is gratefully acknowledged. Much of the research reported was conducted at the University of Illinois at Chicago.

Address correspondence to Dr. Albert B. Schultz, Department of Mechanical Engineering and Applied Mechanics, University of Michigan, Ann Arbor, Michigan 48109-2125. 
curves. Kane and Moe (3) found that in a sample population of young adults, more than one person in every 750 had been referred to a scoliosis clinic for possible treatment. Kane and Moe classified $72 \%$ of these cases as idiopathic. Among them, females outnumbered males 5 to 1 . On the other hand, Brooks et al. reported that in cases of very mild scoliosis, the female to male ratio is only 1.2:1. Other reports of prevalence rates, sex ratios, and etiologic classifications of scoliosis have tended to agree at least roughly with these statistics.

Scoliosis is a condition defined in mechanical terms, so biomechanical studies are relevant. For some years, our laboratory studied the biomechanics of scoliosis treatment [Schultz (8) reviews some of this work]. A few years ago we decided to explore from a biomechanical viewpoint possible causes of idiopathic scoliosis. The important issue seemed to be-not why does a mild scoliosis arise, but why and in which patients does a mild scoliosis progress? The statistics cited indicate that clinically significant progression occurs in perhaps fewer than one out of every 50 patients. Why does progression occur primarily during adolescence, and why does it occur so much more often in girls?

There is an extensive literature inquiring into the causes of idiopathic scoliosis. A comprehensive review is provided by Nachemson and Sahlstrand (5). However, most of the studies cited deal with differences observed between structurally normal subjects and patients with advanced cases of scoliosis. Differences in spine tissue biochemistry, trunk muscle composition and structure, and trunk muscle myoelectric activities, for example, have been reported many times. Such differences offer few clues as to why a mild scoliosis might progress. The differences were observed after significant progression had already occurred, and may well represent secondary effects of the trunk deformities. Studies of differences between structurally normal subjects and subjects with only mild cases of scoliosis are more likely to provide clues as to what causes progression.

This review will concentrate almost exclusively on the research of this kind we have done to try to answer the questions raised above. Few others have thus far attempted comprehensive studies along these lines. The experimental studies made will be reviewed first; then analyses of the biomechanics involved will be reviewed. Finally, current hypotheses of progression mechanics will be discussed.

One can liken the progression of a scoliosis curve in the spine to the gradual buckling of an elastic beam column. Column buckling theory then suggests what biomechanical factors are likely to be of importance to progression. Progression should be more likely to occur if the beam column that the spine constitutes is abnormally slender, is abnormally flexible, or is subjected to abnormal sets of loads.

\section{SPINE SLENDERNESS AND FLEXIBILITY}

Schultz, Sorenson, and Andersson (9) explored the possible role of spine slenderness and Mattson et al. (4) explored the possible role of spine flexi- 
bility in the progression of idiopathic scoliosis. Slenderness (ratios of spine lengths to spine cross-sectional dimensions) was measured from spine roentgenographs of 105 boys and 143 girls with structurally normal spines, and of 21 boys and 219 girls with moderate degrees of idiopathic scoliosis. Spine flexibility was measured from clinical tests of lateral bending abilities in 65 structurally normal girls and 51 girls with moderate degrees of untreated idiopathic scoliosis.

The slenderness data indicated that the girls had spines that were significantly ( $p<0.01$ at ages 12 through 15 ) more slender than those of the boys. When slenderness in the structurally normal girls and the girls with scoliosis (the patient data have not yet been published) was contrasted, some differences appeared, but were not consistent. At best, they only bordered on statistical significance. Spine slenderness, perhaps, can help explain sex differences in progression tendencies, but other factors must also have a role in progression mechanics.

The flexibility data indicated that the structurally normal girls had significantly $(p<0.05)$ more lateral bending flexibility than did the girls with scoliosis. Ability to perform the clinical bending tests probably depended substantially on the true lateral flexibility of the spine. If it did, then this finding tends to controvert hypotheses that idiopathic scoliosis results from excessive flexibility in the soft connective tissues of the spine. Further arguments in support of this reasoning will be given subsequently.

These findings suggested that neither spine slenderness nor spine flexibility have dominant roles in determining whether a mild idiopathic lateral curve in the spine will become more severe.

\section{STRENGTHS OF THE TRUNK MUSCLES}

Unilateral weaknesses or other abnormalities in the muscle of the trunk have often been said to be a cause of idiopathic scoliosis [Nachemson and Sahlstrand (5), for example, provide citations]. Measurements of maximum voluntary trunk strengths provide an easy method to help examine this hypothesis. Portillo et al. (6) measured maximum voluntary isometric trunk strengths in 109 girls with structurally-normal spines and 48 girls with idiopathic scoliosis. Subject ages ranged from 10 to 16 years, with a mean age of approximately 13.5 years in both groups. The girls with scoliosis had lateral curves with a mean Cobb (severity) measure of 21.3 degrees. Twenty seven of these girls were as yet untreated, and 21 were being treated with a brace.

The results showed no consistent differences in mean strengths between the structurally normal trunks and the trunks of patients with idiopathic scoliosis. This was true for all of the trunk strengths measured, and for all trunk-strength ratios computed intra-individually. Neither were there any consistent differences in strengths between the untreated patients and the braced patients. The tests provided no evidence that the strengths of any of the major muscles of the trunk are different in populations of girls with idiopathic scoliosis and age-matched population of girls with structurally normal spines. 
These findings suggest that trunk muscle weakness is not a cause of progression of idiopathic scoliosis. They tend to confirm casual clinical impressions that children with scoliosis have no obvious deficiencies in their physical capabilities.

Portillo et al. found mean maximum strengths in attempted trunk lateral bending of approximately $40 \mathrm{Nm}$ in girls at age 13, for example. Adult lumbar motion segment property data [Schultz et al. (10), for example] show that it requires in the mean approximately a $1 \mathrm{Nm}$ lateral moment to produce a lateral tilt of one degree. These numbers will be referred to subsequently.

\section{MYOELECTRIC ACTIVITY IN THE TRUNK MUSCLES}

Muscles contract in response to electrical signals transmitted by the nerves. Under appropriate circumstances, the magnitudes of these signals (myoelectric activity) are indicative of the muscle contraction forces.

Asymmetric trunk-muscle myoelectric activity has been reported in girls with idiopathic scoliosis by many investigators [Reuber et al. (7) cite and discuss this literature.] To examine this phenomenon in greater depth, Reuber et al. studied trunk-muscle myoelectric activity in biomechanically well-defined experiments. They studied 12 girls with structurally normal spines and 20 female patients with mild-to-moderate idiopathic scoliosis.

Subject ages ranged from 10 to 15 years, with a mean age of approximately 13.5 years in both groups. Patients had lateral curves with a mean Cobb measure of 23.7 degrees. Bipolar surface-electrode pairs picked up activity in the erector spinae and the latissimus dorsi on both sides at the T9 level; and in the erector spinae, the rectus abdominus, and each of the lateral portions and the anteromedial portions of the abdominal oblique muscles on both sides at approximately the L3 level. Each girl performed 15 10-second duration isometric tasks while standing.

Both groups showed significant individual variations in myoelectric signal amplitudes and degree of lateral asymmetry in these signals during task performance. Patients with idiopathic scoliosis with curves of more than 25 degrees had myoelectric signals that were significantly more asymmetric than were those of the girls with structurally normal spines $(p<.01)$. Lumbar level myoelectric signals were higher on the convex sides in the erector spinae muscles while resisting a trunk flexion moment. They were higher in the external abdominal oblique and the rectus abdominus muscles while resisting a lateral bending moment. No other statistically significant differences in myoelectric activities between the normal subjects and scoliotic girls were found. Myoelectric activities in patients whose lateral curves progressed within the year either before or after testing were not significantly different from activities in patients who did not progress. Patients with documented progression who had curves of 25 degrees or fewer showed no significant difference in myoeolectric activities from those of the girls with structurally-normal spines.

Moderately asymmetric trunk-muscle myoelectric activity occurs in girls 
who have structurally-normal spines as well as in girls who have scoliosis. Substantial asymmetries in trunk-muscle myoelectric activity arise only in patients with curves greater than 25 degrees, suggesting that they result from, rather than help create, those lateral curves.

\section{BIOMECHANICAL ANALYSES OF TRUNK MUSCLE ACTIONS}

Haderspeck and Schultz (2) examined quantitatively the effects on spine lateral curves of hypothetical abnormal loads. Such loads might arise from actions of the trunk muscles or malfunctions in the mechanisms used to maintain upright posture of the trunk. The examination was conducted via computer simulation of scoliosis spine biomechanics (Fig. 1). For this simulation, the bones of the trunk were represented as rigid bodies, and the passive soft tissues interconnecting them were represented as linear elastic deformable elements. Muscle contractions were represented by applying pairs of equal and opposite known forces to each two of a series of nodes lying along the trunk muscle lines-of-action. In this way, the immediate deformations of the trunk that resulted from the contractions of given muscles or use of different bodyweight support strategies could be studied. The influences of lateral and anteroposterior curve morphologies, lateral curve severities, trunk muscle contraction force levels, and body weight support strategies on these deformations were investigated.

The rationale for this approach, given by Haderspeck and Schultz, was that the abnormal loads of concern in scoliosis must be unbalanced spine lateral bending moments. Compression alone on a motion segment does not cause a significant lateral tilt. When unbalanced lateral moments are imposed on a spine, its involved motion segments will take on lateral tilts. They will tilt laterally enough for them to equilibrate the unbalanced moments, through the deformation and consequent stressing of their soft-tissue constituents. These lateral tilts contribute to changes in the Cobb measure. Suppose that even only small imbalances in lateral bending moments must be continually resisted by lateral tilts of the spine motion segments in vivo. Then soft tissue growth and remodeling processes eventually must translate these small lateral tilts into the large lateral structural deformities seen in the advanced stages of scoliosis. So, the immediate lateral tilts caused by a muscle contraction or a body weight support strategy are probably indicative of the long-term progression tendency that continued contractions of that muscle or continual use of that strategy would produce.

Some of the salient findings of the study were: (1) At modest contraction intensities, unilateral contractions of some trunk muscles spanning the concave side of a lateral curve can cause substantial Cobb measure (severity) increases. The internal abdominal obliques and the erector spinae can substantially increase a lumbar curve; the latissimus, intercostals, and erector spinae can substantially increase a thoracic curve. (2) Contractions of the 


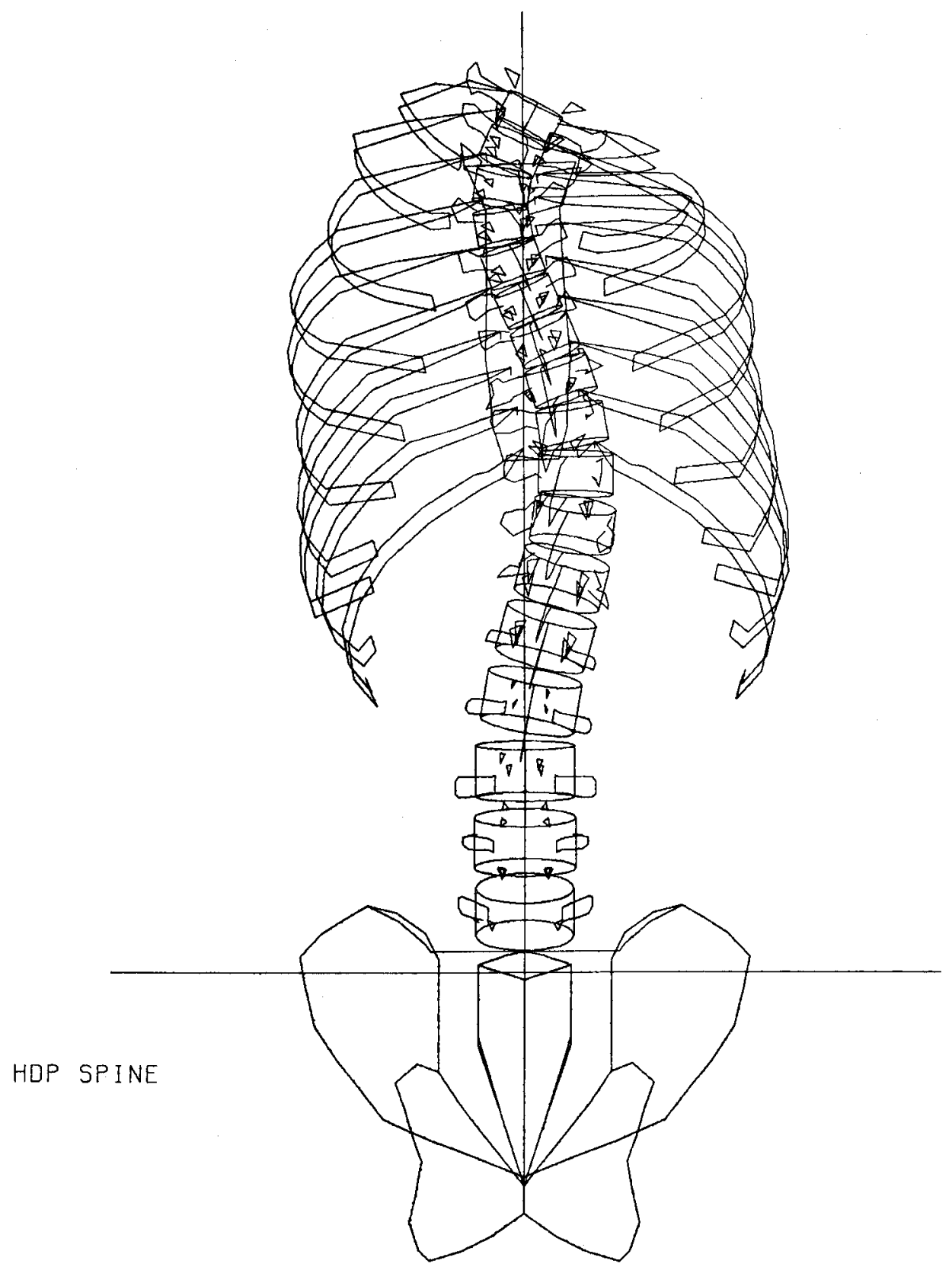

FIGURE 1. Computer-simulated biomechanical model of a spine with scoliosis.

additional muscles needed to maintain the trunk upright significantly alter the effects of an initial trunk muscle contraction. (3) The effect of a trunk muscle contraction on a lateral curve is not influenced very much by the presence of other lateral curves or by other differences in spine configurations. 
(4) Application of the weights of upper body segments to a laterally curved spine can cause significant curve increases. The amounts of these increases depend on the initial spine configuration and the nature of trunk-righting responses to the weight application.

In relaxed upright postures of the trunk in the structurally normal spines of individuals, spine motion segments probably are seldom called upon to resist lateral bending moments through lateral tilting. The neural mechanisms controlling trunk posture must sense the existence of any nonzero moments on the spine and signal the trunk muscles accordingly. The trunk muscles must then contract appropriately to bring the spine lateral moments back to zero.

Unbalanced spine lateral bending moments probably arise from inability of the neural mechanisms to sense a spine moment imbalance or to direct the needed response properly. Suppose that the spine motion segment zero-lateraltilt signals are in error. The studies showed that almost any one of the trunk muscles has the capability to produce in response the few Newton meters of lateral moment imbalance presumably needed to promote curve progression. The studies showed that postural offsets of upper body segment weights of several different kinds can promote lateral curve increases of several different natures. The studies suggested that details of postural configuration inferior to the sacrum are not in themselves directly relevant to lateral curve progression mechanisms. Finally, the studies showed that righting mechanism selection can have significant influences on lateral curve progression tendencies. The interpretations given to these model study findings by Haderspeck and Schultz were recently reexamined and modified by Reuber et al. (7). This will be described in the next section.

Consider again the question of whether abnormal soft tissue flexibilities might be involved in progression mechanics. The biomechanical model study just cited only considered possible roles of unbalanced spine lateral bending moments in the progression of lateral curves. It did not consider the effects of changes in soft-tissue properties, perhaps due to hormonal or other biochemical influences. The relevance of such changes to the progression of scoliosis is suggested by the many observations that progression rarely occurs except during the rapid growth period of adolescence. On the other hand, biomechanical analyses bring out that passive resistances of spine motion segments to the small lateral tilts involved in a mild lateral curve are, at most, a few Newton meters. However, the trunk muscles, even in adolescent girls, can generate lateral bending moments of the order of $40 \mathrm{Nm}$. So, soft-tissue resistance changes, even by large factors, should still have little effect on the responses of the spine in lateral bending. If tissue-property changes were involved in scoliosis, the likely ones would be those that make the motion segments more laterally flexible. To bring the lateral tilt of a normal-motion segment to zero is a task that should not tax the trunk muscles; the task should be even easier if the motion segment is abnormally flexible. These are further arguments that soft-tissue flexibility differences are not primarily involved in scoliosis progression. 


\section{CURRENT HYPOTHESES CONCERNING PROGRESSION BIOMECHANICS}

In overall summary of the studies reviewed so far, hypotheses that the source of progression of idiopathic scoliosis lies in defects in the neural systems that control upright postures of the trunk seem attractive. They seem more consistent with observed facts and the findings of biomechanical analyses than do other hypotheses about progression sources. These other hypotheses include excessive spine slenderness, excessive spine tissue flexibility, and decreased mechanical capabilities of the trunk muscles. These other hypotheses cannot be ruled out, but current evidence points away from them. Based upon what had been learned from the studies described, Reuber et al. (7) constructed more specific hypotheses about progression biomechanics in idiopathic scoliosis due to neural control defects.

Once a curve to the side exists in a spine, the weight of the body segments superior to that curve in upright positions of the trunk creates a lateral bending moment that tends to increase it. This follows from simple biomechanical analysis (Fig. 2). The weight involved is of the order of $200 \mathrm{~N}$, and the lateral offset of the apical vertebra in a mild curve is probably at least $2.5 \mathrm{~mm}$, so the moment created is on the order of $0.5 \mathrm{Nm}$. So, once a small curve to the side exists, a moment tending to increase it is potentially present whenever the trunk is upright. Note that this moment is only about $1 \%$ of the mean lateral muscular strength moment that can be developed by an adolescent girl.

Based on this realization, Reuber et al. proposed two hypotheses. First, progression of a scoliosis impends when lateral asymmetry in trunk-muscle contraction forces is too small to balance the lateral bending moments produced by superior body segment weights acting on the laterally offset vertebrae in the curve. In other words, Haderspeck and Schultz (2) had argued that the trunk muscles easily had the capability to promote curve progression through active contractions. If posture control system malfunctions instructed the muscles to create unbalanced lateral bending moments, they could do so with little effort. Reuber et al. argued for a passive mechanism for progression; the unbalanced moment is potentially present, and it is the failure of the control system to respond that promotes progression. If the contraction forces provided by the motor control system are too laterally symmetric, it falls to the soft tissues of the motion segments within the curve to supply the resistances necessary to balance the remainder of the lateral bending moments. Those motion segments must tilt further in order to do this. Curve severity increases as a result. This hypothesis seems reasonable as a necessary but perhaps not a sufficient condition for a scoliosis curve to progress.

The second hypothesis of Reuber et al. relates to the long-term response of motion segment soft tissues (principally, those of the intervertebral disc) to unbalanced lateral bending moments. A scoliosis will progress if, in longterm response to the nearly constant lateral bending moments on the intervertebral discs that result from the motor control malfunction, their lateral tilts both continue to increase and become semipermanent. 


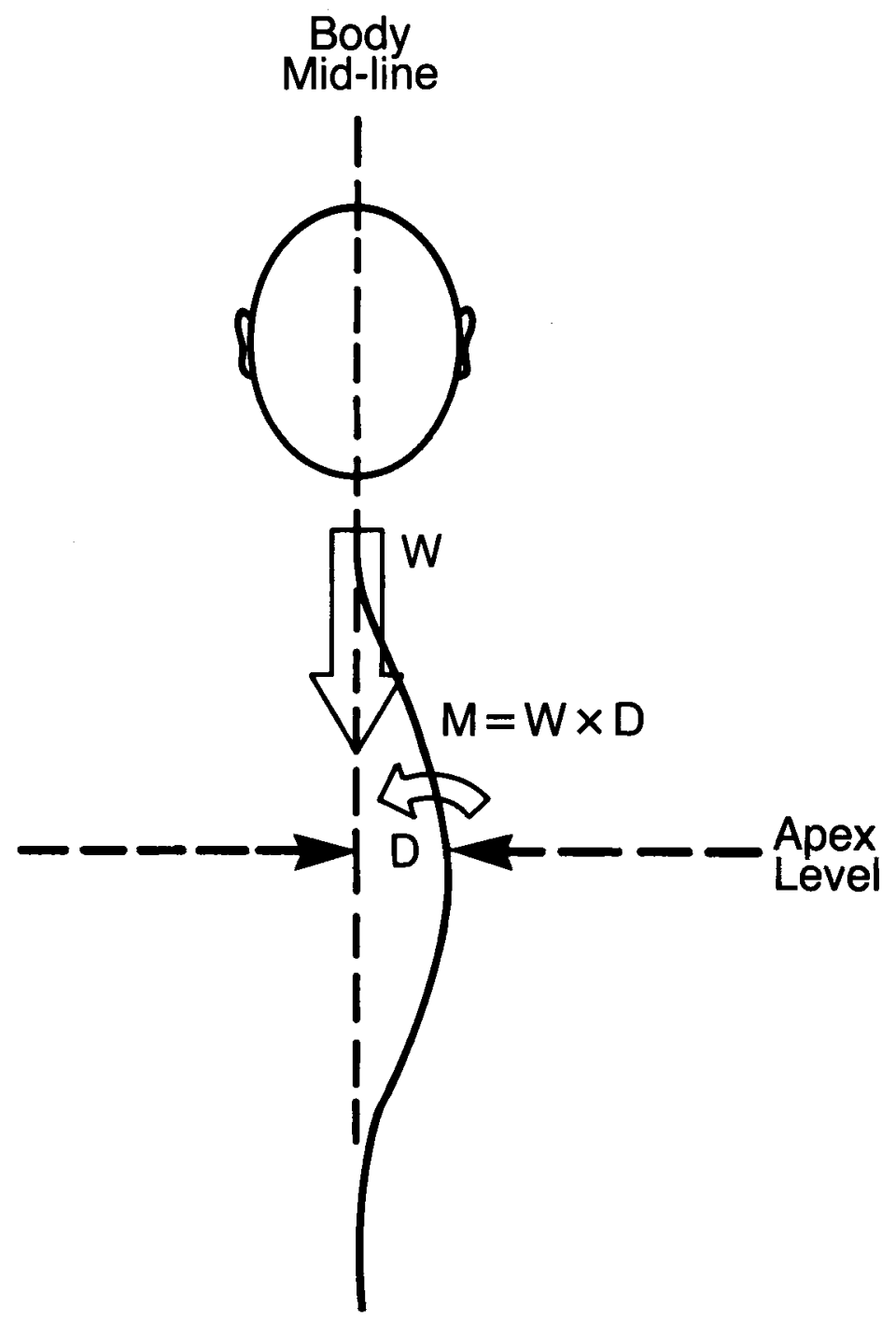

FIGURE 2. Schematic diagram of the moment produced when body weight acts on a laterally-offset spine. $W$ denotes the weight of all body segments above the level of the apex, $D$ denotes the lateral translation of the apical vertebra from the body midline, and $M$ denotes the lateral bending moment at the apex that results from this lateral offset. From Reuber et al. (7).

A number of investigations have shown that when spines of structurally normal animals are held in laterally curved positions for several weeks, the lateral tilts of the vertebrae become semipermanent. Research into the properties of the soft tissues of the spine in patients with scoliosis might examine tendencies to continue to tilt (that is, to creep) and to develop semipermanent tilts under sustained small-lateral bending moments, rather than shortterm lateral bending stiffness properties.

Why does idiopathic scoliosis tend to progress during the adolescent growth 
spurt, and why does it progress so much more often in girls than in boys? The answers to these questions remain unknown. The hypothetical necessary condition for progression-subtle malfunctioning of the motor control system - may arise only during maturation of that system, and it may arise much more often in girls than in boys. On the other hand, slightly inappropriate symmetry control may be present at all ages and perhaps equally prevalent in the sexes. It would then be the tendency of disc lateral tilts to increase and become semipermanent which would dominate the tendency for progression. Perhaps these tendencies become significant principally during the adolescent growth spurt, and primarily in girls.

These ideas need to be explored in depth. Nevertheless, the two hypotheses of Reuber et al. seem consistent, both qualitatively and quantitatively, with what is presently known about the biomechanics of progression of idiopathic scoliosis.

\section{REFERENCES}

1. Brooks, H.L., S.P. Azen, E. Gerberg, R. Brooks, and L. Chan. Scoliosis: A prospective epidemiological study. J. Bone Jt. Surg. 57A:968-972, 1975.

2. Haderspeck, K. and A. Schultz. Progression of idiopathic scoliosis: An analysis of muscle actions and body weight influences. Spine 6:447-455, 1981.

3. Kane, W. and J. Moe. A scoliosis-prevalence survey in Minnesota. Clin. Orthop. 69:216-218, 1970.

4. Mattson, G., K. Haderspeck-Grib, A. Schultz, and A. Nachemson. Joint flexibilities in structurallynormal girls and girls with idiopathic scoliosis. J. Orthop. Res. 1:57-62, 1983.

5. Nachemson, A. and T. Sahlstrand. Etiologic factors in adolescent idiopathic scoliosis. Spine 2:176-184, 1977.

6. Portillo, D., G. Sinkora, T. McNeill, D. Spencer, and A. Schultz. Trunk strengths in structurallynormal girls and girls with idiopathic scoliosis. Spine 7:551-554, 1982.

7. Reuber, M., A. Schultz, T. McNeill, and D. Spencer. Trunk muscle myoelectric activities in idiopathic scoliosis. Spine 8:447-456, 1983.

8. Schultz, A. A biomechanical view of scoliosis. Spine 1:162-171, 1976.

9. Schultz, A., S. Sorensen, and G. Andersson. Measurements of spine morphology in children, ages 10-16. Spine 9:70-73, 1984.

10. Schultz, A.B., D.N. Warwick, M.H. Berkson, and A.L. Nachemson. Mechanical properties of human lumbar spine motion segments. Part I. Responses in flexion, extension, lateral bending and torsion. J. Biomech. Eng. 101:46-52, 1979.

11. Shands, A.R. and H.B. Eisberg. The incidence of scoliosis in the state of Delaware. J. Bone Jt. Surg. 37A:1243-1248, 1955. 